\title{
HARNESSING POISSON EFFECT TO REALIZE TUNABLE TUNNELING NANOGAP ELECTRODES ON PDMS SUBSTRATES FOR STRAIN SENSING
}

\author{
Henry S.C. Yu*, Giovanni Boero, and Jürgen Brugger \\ Microsystems Laboratory, Institute of Microengineering, School of Engineering, \\ École Polytechnique Fédérale de Lausanne (EPFL), 1015 Lausanne, SWITZERLAND
}

\begin{abstract}
Nanogap electrodes (NGEs) are a fundamental structure for the exploration of nanoscale phenomena. In this paper, we describe the fabrication and the electro-mechanical characterization of tunable tunneling NGEs on a stretchable PDMS substrate. Bottom-up capillary-assisted particle assembly (CAPA) in nano-scale traps is used to fabricate Au nanorod dimers as NGEs. The nanogap is tuned by the contraction due to the Poisson effect of the PDMS substrate. Finite element method (FEM) simulations are performed to quantify how the NGEs design affects the local Poisson contraction under applied tensile strain. The application of the proposed approach for strain sensing is discussed.
\end{abstract}

\section{KEYWORDS}

Tunable nanogap electrodes, stretchable, tunneling effect, Poisson effect, capillary-assisted particle assembly, strain sensing.

\section{INTRODUCTION}

NGEs comprise a pair of electrically conducting materials separated by an nm-scale air gap. Over past decades, NGEs have been utilized in applications such as molecular electronics [1], nanoantennas [2], DNA sequencing [3], and nanoelectromechanical switches [4]. For nanogaps $<3 \mathrm{~nm}$ (so-called tunneling regime), the exponential dependence of the tunneling current on the gap width makes NGEs potentially interesting for cases where the tunneling gap is tunable on a stretchable substrate. Up to now, however, such devices have not been reported, as they are challenging to be fabricated reliably. Liu et al. [5] demonstrated mechanically tunable sub-10 nm metal gap by stretching PDMS substrate for tunable plasmonics. The tunability of the metal gap distance was verified by optical spectra rather than electrical measurements. The same group in 2018 [6] used pre-strained PDMS substrate during the metal structure transfer to demonstrate the precise control of the tunable nanogap from $108 \mathrm{~nm}$ to sub-10 nm, but the tunneling regime was not demonstrated.

Randomly separated nanogaps formed by assembled nanoparticles were used for strain sensing [7]. Conducting tunneling paths between nanoparticles feature high gauge factor $(>730)$ but it was only demonstrated for strain $<1 \%$ due to the tunneling cut-off. Such a trade-off between sensitivity and stretchability is a common issue in strain sensing techniques [8]. Here we describe a possible alternative approach, which exploits the Poisson effect and the engineering of NGEs for strain sensing with tunable stretchability range.

\section{Contributions}

- The utilization of bottom-up CAPA to fabricate
NGEs on a stretchable substrate

- Harnessing the Poisson effect to realize tunable tunneling NGEs

- $\quad$ FEM simulations to show a potential application in stretchable strain sensors

\section{DEVICE DESCRIPTION}

(a) Initial state

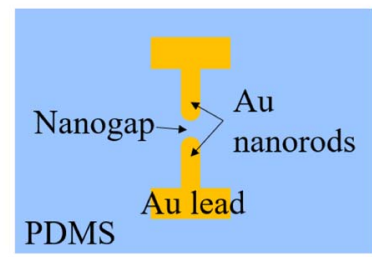

(b) Strain applied

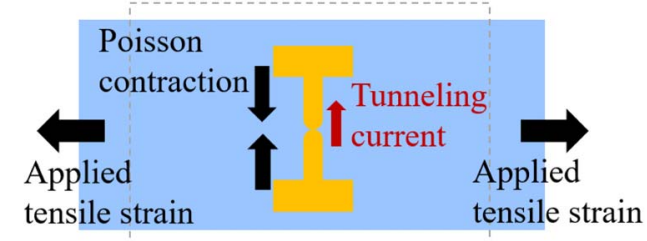

Figure. 1: Illustration of the working principle of the tunable tunneling NGEs. (a) Initial state (zero strain) (b) under tensile strain

Fig. 1 shows the working principle of the tunable NGEs. In this configuration, the nanogap changes when applying a tensile force orthogonal to the $\mathrm{Au}$ nanorod dimer due to Poisson contraction in the longitudinal direction. At the initial state, no strain is applied and the two nanorods are separated by an initial nanogap. When a tensile strain is applied on the PDMS substrate, the two nanorods are moving closer to each other, and the tunneling current can be measured by applying a DC voltage across the NGEs.

\section{MODELING AND EXPERIMENTATION Simulation model}

COMSOL, a standard FEM software, is used to perform simulations to quantify how the NGEs dimensions affect the local Poisson contraction under applied tensile strain. As shown in Fig. 2, a pair of $150 \mathrm{~nm}$ thick Au leads is placed at the center of PDMS in the $\mathrm{x}$ and $\mathrm{y}$ directions. In the $\mathrm{z}$-direction, the Au leads are embedded in PDMS with their top surfaces being in the same $x-y$ plane. The PDMS initial dimensions are $15 \mathrm{~mm}, 7 \mathrm{~mm}$, and $1 \mathrm{~mm}$ in the $\mathrm{x}, \mathrm{y}$, and $\mathrm{z}$ directions respectively. The initial $\mathrm{Au}$ leads spacing $d_{0}$ is set to be $0.5 \mu \mathrm{m}$. Different lengths and widths of $\mathrm{Au}$ leads are simulated to calculate the displacement field in y-direction under tensile strain $\varepsilon$ applied on the PDMS 
substrate in the $\mathrm{x}$-direction. The resulting Poisson contraction of the Au leads spacing $\Delta d$ in the y-direction is then used to calculate the local Poisson's ratio $v_{\text {local }}$ by:

$$
v_{\text {local }}=-\left(\Delta d / d_{0}\right) / \varepsilon
$$

In the COMSOL solid mechanics model, geometric nonlinearity is included. Both, $\mathrm{Au}$ and PDMS are considered as linear elastic materials since the applied tensile strain is not larger than $5 \%$.

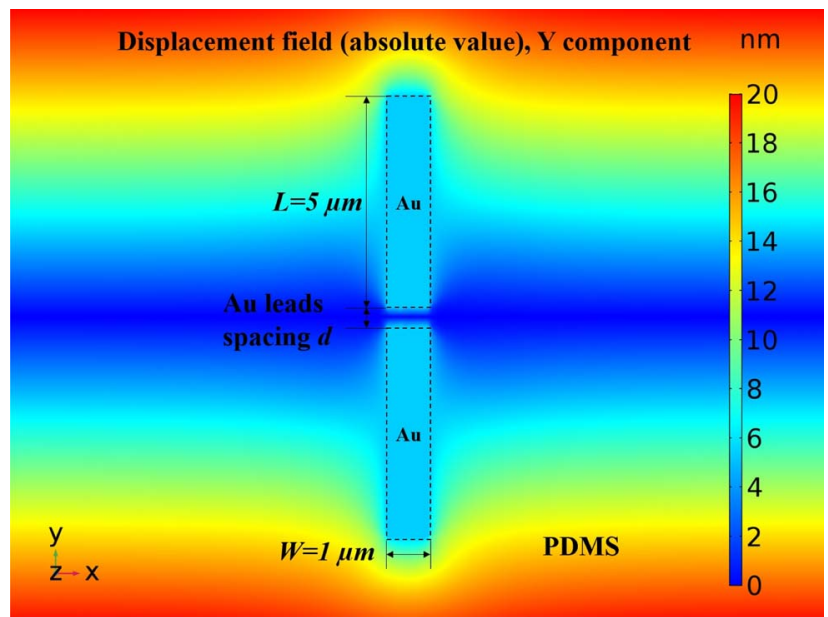

Figure 2: Contour map of the displacement field (absolute value) in y-direction simulated by COMSOL.

(1) Traps formation \&

$\mathrm{Al}$ surface treatment

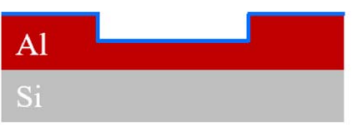

(2) Capillary-assisted particle assembly (CAPA)

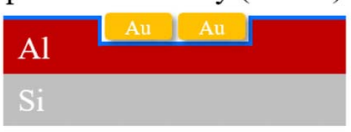

(3) Au leads formation

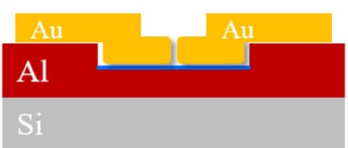

(5) Sacrificial Al wet etch for structures transfer

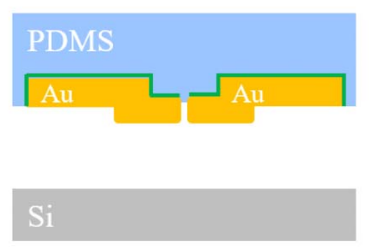

Figure 3: Fabrication process flow (cross-sectional view).

\section{Fabrication}

Fig. 3 shows the fabrication processes of the tunable tunneling NGEs. A Ti $(10 \mathrm{~nm}) / \mathrm{Al}(300 \mathrm{~nm})$ thin film serving as a sacrificial layer is deposited on a silicon substrate by e-beam evaporation deposition. The in-situ Ti thin film is deposited prior to $\mathrm{Al}$ in order to have a smooth Al thin film surface $\left(R_{a}\right.$ and $\left.R_{r m s}<10 \mathrm{~nm}\right)$. E-beam lithography and reactive ion etch (RIE) are then used in step (1) to pattern the $\mathrm{Al}$ traps with dimensions $\mathrm{L} / \mathrm{W} / \mathrm{D}=$ $2600 \mathrm{~nm} / 200 \mathrm{~nm} / 100 \mathrm{~nm}$. After photoresist removal, $\mathrm{O}_{2}$ plasma surface activation and vapor phase silanization with Trichloro(1H,1H,2H,2H-perfluorooctyl)silane (abcr
$\mathrm{GmbH}$ ) are conducted to make Al surface hydrophobic for the following assembly process (DI water contact angle of about $103^{\circ}$ ). In step (2), Au nanorods (Nanopartz Inc., L: $1000 \mathrm{~nm}$, D: $100 \mathrm{~nm}$ ) are assembled using CAPA [9] as shown in Fig. 4. When the moving meniscus passes over predefined traps, the capillary force inserts the $\mathrm{Au}$ nanorods into the traps while dragging away excessive nanorods on the hydrophobic silanized Al surface. The drying of the residual solvent in the traps brings the two nanorods close together. In an extreme case, they are separated only by the ligand molecule (CTAB) on the $\mathrm{Au}$ surface. $\mathrm{O}_{2}$ plasma treatment after CAPA removes CTAB and silane on the $\mathrm{Au}$ and $\mathrm{Al}$ surfaces, respectively. In step (3), the lift-off process of e-beam lithography followed by the $150 \mathrm{~nm}$ Au e-beam evaporation deposition defines the Au leads and pads connecting to the assembled Au nanorod dimer. In step (4), the sample is firstly immersed in $60 \mathrm{mM}$ MPTMS ethanol solution ((3-Mercaptopropyl)trimethoxy silane, Sigma Aldrich) for 2 hours to modify the Au surface in order to enhance the adhesion between Au and PDMS. After isopropanol rinse and air blow dry, $1 \mathrm{~mm}$ thick 10:1 PDMS is poured on the sample and cured at room temperature for at least 48 hours to minimize any thermal stress. Finally, the sacrificial $\mathrm{Al}$ layer is removed by exposing the $2 \times 2 \mathrm{~mm}^{2}$ sample to a solution of $37 \%$ hydrochloric acid : DI water $=1: 6$ for about 7 hours, followed by DI water cleaning.

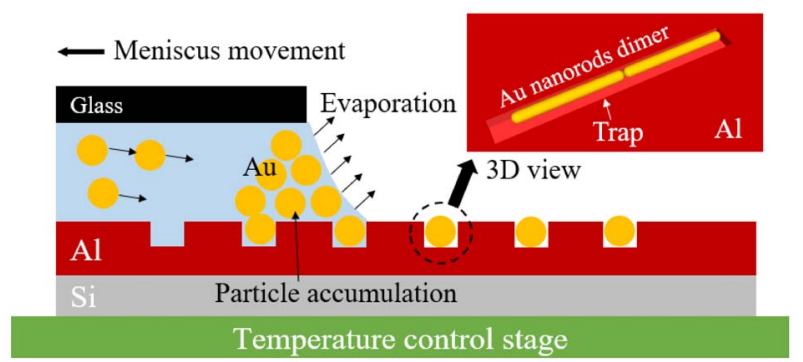

Figure 4: Illustration of the bottom-up CAPA process.

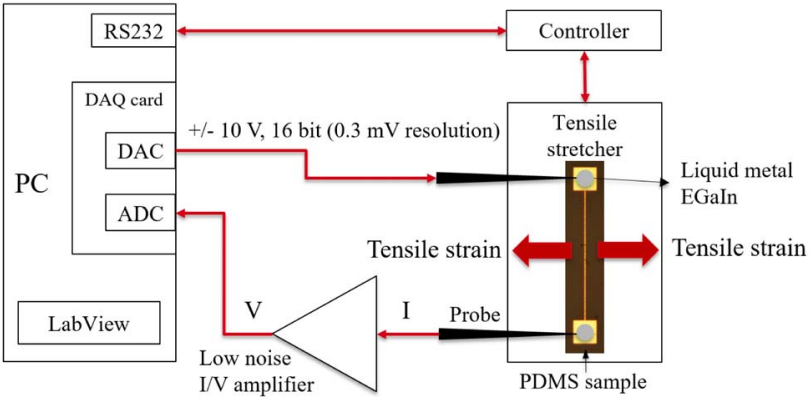

Figure 5: Schematic view of the measurement setup.

\section{Measurement setup}

Fig. 5 shows the measurement setup with the PDMS sample with the Au NGEs being mechanically clamped in a stretcher (TST350, Linkam Scientific Ltd.). Eutectic $\mathrm{Ga} / \mathrm{In}$ liquid metal (Sigma-Aldrich) is added on the $\mathrm{Au}$ pads to ensure a reliable contact between the probing needles and the Au pads during measurements. One of the probing needles is connected to a data acquisition (DAQ) card (PCIe-6363, National Instruments), whereas the other probing needle is connected to a low noise $\mathrm{I} / \mathrm{V}$ amplifier 
(SR570, Stanford Research Systems Inc.), which converts current signals into voltage signals. The output and input voltage signals are applied and detected by the DAQ card, which is controlled by a program written in LabView (National Instruments).

\section{RESULTS \\ FEM simulation}

FEM simulation results in Table 1 show that when the length of Au leads is increased from $5 \mu \mathrm{m}$ to $500 \mu \mathrm{m}$ with a fixed width of $1 \mu \mathrm{m}$, the local Poisson's ratio increases significantly from 4.3 to 307.2 . This means that under the same applied strain, the contraction of PDMS between $\mathrm{Au}$ leads is larger for longer Au leads. It can be understood as the fact that when a larger area of PDMS in the longitudinal direction is "stiffened" by longer Au leads, the PDMS area not affected by the Au layer contracts more to absorb the applied work as the strain energy. On the other hand, as the width of $\mathrm{Au}$ leads is increased from $1 \mu \mathrm{m}$ to $10 \mu \mathrm{m}$ with the length fixed at $5 \mu \mathrm{m}$, the local Poisson's ratio only slightly drops from 4.3 to 2.4. As a result, the local Poisson's ratio can be configured by changing the lateral dimensions of the Au leads.

Table 1: COMSOL FEM results. A pair of Au leads on PDMS separated by an initial spacing of $0.5 \mu \mathrm{m}$ in the longitudinal direction to the applied tensile strain is considered (without nanorods). The Poisson' ratio is calculated by Poisson contraction / applied tensile strain.

\begin{tabular}{|r|r|r|r|r|r|r|}
\hline \multicolumn{4}{|c|}{ Inputs } & \multicolumn{3}{c|}{ Outputs } \\
\hline $\begin{array}{r}\text { Length leads } \\
(\boldsymbol{\mu m})\end{array}$ & $\begin{array}{l}\text { Width } \\
(\boldsymbol{\mu} \mathbf{m})\end{array}$ & $\begin{array}{l}\text { Initial } \\
\text { spacing } \\
(\boldsymbol{\mu} \mathbf{m})\end{array}$ & $\begin{array}{l}\text { Applied } \\
\text { tensile } \\
\text { strain }\end{array}$ & $\begin{array}{l}\text { Au lead } \\
\text { spacing } \\
\text { variation } \\
(\mathbf{n m})\end{array}$ & $\begin{array}{l}\text { Poisson } \\
\text { contraction }\end{array}$ & $\begin{array}{l}\text { Poisson's } \\
\text { ratio }\end{array}$ \\
\hline 500 & 1 & 0.5 & $0.01 \%$ & 15 & $3.1 \%$ & 307.2 \\
\hline 50 & 1 & 0.5 & $0.05 \%$ & 11 & $2.2 \%$ & 44.8 \\
\hline 5 & 1 & 0.5 & $0.5 \%$ & 11 & $2.1 \%$ & 4.3 \\
\hline 5 & 5 & 0.5 & $0.5 \%$ & 7 & $1.4 \%$ & 2.9 \\
\hline 5 & 10 & 0.5 & $0.5 \%$ & 6 & $1.2 \%$ & 2.4 \\
\hline
\end{tabular}

\section{Fabrication}

Fig. 6 depicts the result of the tunable tunneling NGEs fabrication. The top of the figure shows the optical microscope image of the PDMS sample at the end of the fabrication process. Both, the $\mathrm{Au}$ leads and pads are successfully transferred from the silicon substrate to the PDMS substrate. Au pads and leads as small as $1 \mu \mathrm{m}$ remain intact due to the minimized mechanical stress during the wet transfer process. Since it is challenging to image the PDMS sample in a scanning electron microscope (SEM) with high resolution, the SEM image of the nanogap is taken on the silicon substrate prior to the transfer process. As shown at the bottom in Fig. 6, the diameters of the Au nanorods are of about $50 \mathrm{~nm}$, which is smaller than the nominal diameter of $100 \mathrm{~nm}$. The actual dimension of the nanogap cannot be measured accurately because of the resolution limitation of the SEM. Each Au lead comprises of two rectangles: $1 \times 1 \mu \mathrm{m}^{2}$ contacting the nanorod and $5 \times 600$ (left) / 860 (right) $\mu \mathrm{m}^{2}$ in connection with the pad. The Au leads have a thickness of $150 \mathrm{~nm}$.

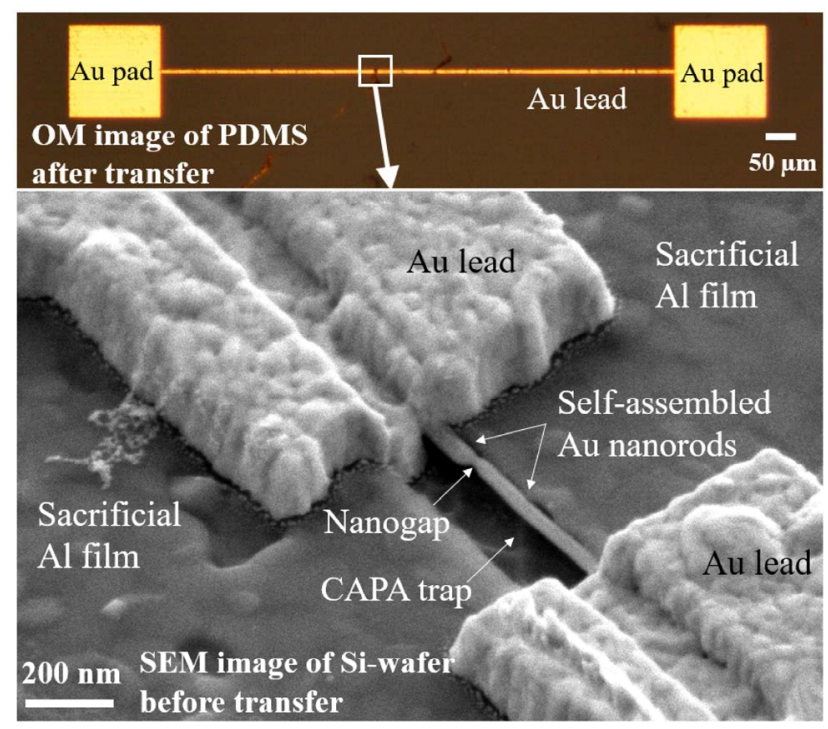

Figure 6: The optical microscope image (top) shows the device after transfer to the PDMS support; the SEM image (bottom) shows the NGEs on the silicon wafer before transfer (Scale bars: $50 \mu \mathrm{m}$ and $200 \mathrm{~nm}$ respectively).
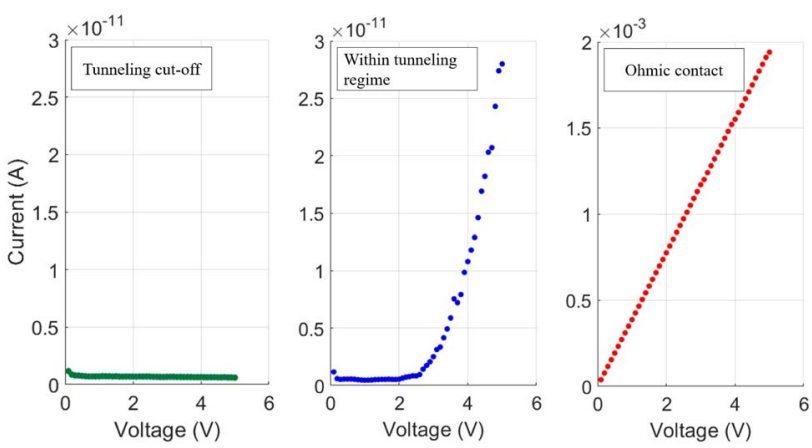

Figure 7: I-V curves of NGEs under different applied static tensile strain.

\section{Tunable tunneling NGEs}

According to FEM simulation results in Table 1, the nanogap is expected to be in tunneling regime under the applied strain smaller than $0.01 \%$. During the measurement, however, tunneling current is not detected until the applied strain is $2 \%$ to $5 \%$. This could be explained by the strain control at the initial status. As the PDMS slab is fixed in the stretcher, a tensile strain $>0.01 \%$ could be already applied to the PDMS. Fig. 7 shows three $\mathrm{I}-\mathrm{V}$ curves under different strain levels. At initial status, the nanogap is larger than the tunneling cut-off distance and only a leakage current of less than $1 \mathrm{pA}$ is measured. Increasing the strain to $2 \%$ to $5 \%$, the nanogap between $\mathrm{Au}$ nanorods is narrowed down and the tunneling current is measured. This is confirmed by the Fowler-Nordheim representation of the same I-V data as shown in Fig. 8. The direct tunneling is shown as $1 / \mathrm{V}$ is larger than 0.5 , whereas the linear curve as $1 / \mathrm{V}$ is smaller than 0.5 signifies the F-N field emission tunneling. The F-N tunneling dominates the electrical conduction when the applied DC voltage $V>\Phi_{B}$ $/ e$, where $\Phi_{B}$ is the potential barrier height of the $\mathrm{Au}$ nanogap and $e$ is the electron charge. According to Fig. 8, $\Phi_{B}$ is estimated $\approx 2 \mathrm{eV}$ in this case, which agrees well with previously published results $[2,10]$. As the applied strain 
further increases, a linear I-V curve is measured, indicating the Au nanorods are in Ohmic contact $(\sim 2.6 \mathrm{kOhm})$.

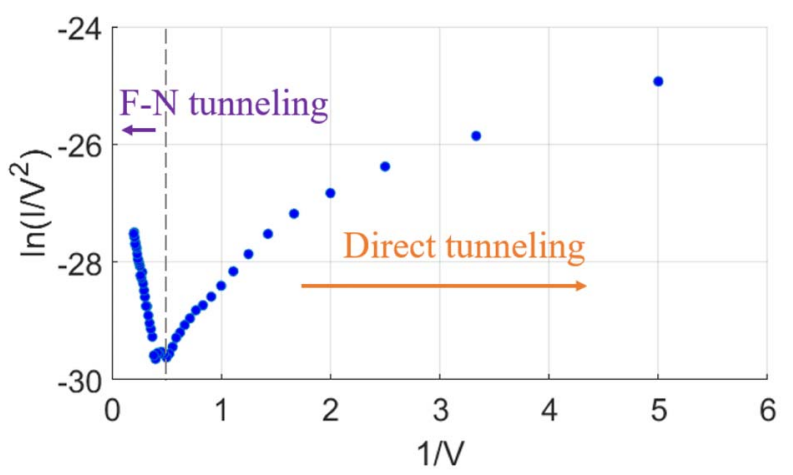

Figure 8: The Fowler-Nordheim representation of the same I-V data in Fig. 7 (in blue).

\section{Potential application in strain sensing}

Further FEM simulation as shown in Fig. 9 suggests the possibility to further reduce the local Poisson's ratio. By adding dummy $\mathrm{Au}$ with a $50 \mathrm{~nm}$ spacing to the $\mathrm{Au}$ leads, the Au leads spacing contracts from $0.5 \mu \mathrm{m}$ to 0.496 $\mu \mathrm{m}$ under $5 \%$ applied strain. The local Poisson's ratio is 0.17 . Thereby, for the application of strain sensing, increasing the stretchability of NGEs by configuring the local Poisson's ratio is achievable by optimizing the material layout around the nanogap.

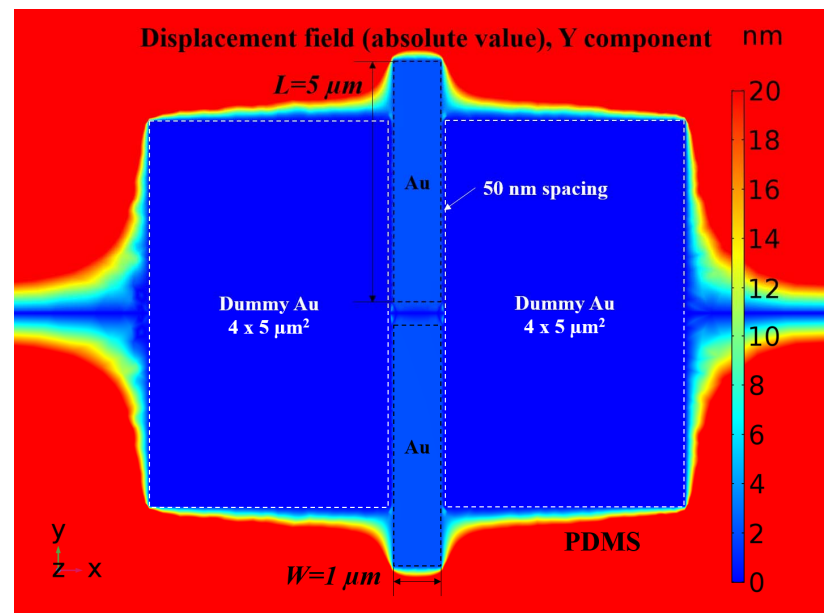

Figure 9: Contour map of the displacement field (absolute value) in y-direction simulated by COMSOL for a design with dummy $\mathrm{Au}$.

\section{CONCLUSIONS}

We describe tunable tunneling NGEs on a PDMS substrate by utilizing the Poisson effect. A combined top-down e-beam lithography lift-off process and bottom-up CAPA process are used to fabricate Au nanorod dimers as NGEs. Electro-mechanical characterization of NGEs is conducted to confirm that the nanogap can be tuned between states of open, tunneling, and ohmic contact. Preliminary FEM simulations show that the local Poisson's ratio is significantly affected by the NGEs design such as Au lead dimensions as it locally stiffens the PDMS surface. This illustrates the potential of the tunable tunneling NGEs proposed in this work as a strain sensor.
By careful optimization of the NGEs design, it should be possible to operate in the tunneling regime for the desired stretchability range.

\section{ACKNOWLEDGEMENTS}

This work received funding from the European Research Council (ERC); project MEMS 4.0 "Additive Micro-Manufacturing for Plastic Micro-Electro-Mechanical-Systems". The authors also thank staffs of the Center of MicroNanoTechnology (CMi) in EPFL for expert support of the fabrication processes.

\section{REFERENCES}

[1] M. Tsutsui and M. Taniguchi, "Single molecule electronics and devices," Sensors (Switzerland), vol. 12, no. 6, pp. 7259-7298, 2012.

[2] J. Kern, R. Kullock, J. Prangsma, M. Emmerling, M. Kamp, and B. Hecht, "Electrically driven optical antennas," Nat. Photonics, vol. 9, no. 9, pp. 582-586, 2015.

[3] C. Gu, C. Jia, and X. Guo, "Single-Molecule Electrical Detection with Real-Time Label-Free Capability and Ultrasensitivity," Small Methods, vol. 1, no. 5, p. 1700071, 2017.

[4] J. O. Lee et al., "A sub-1-volt nanoelectromechanical switching device," Nat. Nanotechnol., vol. 8, no. 1, pp. 36-40, 2013.

[5] W. Liu, Y. Shen, G. Xiao, X. She, J. Wang, and C. Jin, "Mechanically tunable sub-10 nm metal gap by stretching PDMS substrate," Nanotechnology, vol. 28, no. 7, 2017.

[6] W. Liu, Q. Zou, C. Zheng, and C. Jin, "Metal-Assisted Transfer Strategy for Construction of 2D and 3D Nanostructures on an Elastic Substrate," ACS Nano, vol. 13, pp. 440-448, 2018.

[7] J. L. Tanner, D. Mousadakos, P. Broutas, S. Chatzandroulis, Y. S. Raptis, and D. Tsoukalas, "Nanoparticle strain sensor," Procedia Eng., vol. 25, pp. 635-638, 2011.

[8] M. Amjadi, K. U. Kyung, I. Park, and M. Sitti, "Stretchable, Skin-Mountable, and Wearable Strain Sensors and Their Potential Applications: A Review," Adv. Funct. Mater., vol. 26, no. 11, pp. 1678-1698, 2016.

[9] V. Flauraud et al., "Nanoscale topographical control of capillary assembly of nanoparticles," Nat. Nanotechnol., vol. 12, no. 1, pp. 73-80, 2016.

[10]S. P. Gurunarayanan et al., "Electrically Driven Unidirectional Optical Nanoantennas," Nano Lett., vol. 17, no. 12, pp. 7433-7439, 2017.

\section{CONTACT}

*Henry S.C. Yu, e-mail: shao-chi.yu@epfl.ch, tel: $+41-21-693-4981$ 